\title{
Early expression of local cytokines during systemic Candida albicans infection in a murine intravenous challenge model
}

\author{
VOON KIN CHIN $^{1}$, KUAN JEANG FOONG ${ }^{1}$, ABDULLAH MAHA $^{2}$, \\ BASIR RUSLIZA $^{3}$, MOHTARRUDIN NORHAFIZAH ${ }^{2}$ and PEI PEI CHONG ${ }^{1,4}$
}

\begin{abstract}
Departments of ${ }^{1}$ Biomedical Science, ${ }^{2}$ Pathology and ${ }^{3}$ Human Anatomy, Faculty of Medicine and Health Sciences, University Putra Malaysia, Serdang, Selangor 43400, Malaysia; ${ }^{4}$ Translational Infectious Diseases Program, Centre for Translational Medicine, Department of Microbiology, National University of Singapore, Singapore 117597, Republic of Singapore
\end{abstract}

Received July 10, 2014; Accepted August 13, 2014

DOI: $10.3892 /$ br.2014.365

\begin{abstract}
Local cytokine production is a significant indicator for disease pathogenesis or progression. Previous studies on cytokine production during systemic Candida albicans (C. albicans) infection were solely on kidney or single cell type interaction with $C$. albicans. Therefore, the present study aimed to assess the early cytokine expression of various target organs (kidney, spleen and brain) over a 72-h time course during systemic $C$. albicans infection. The local cytokine profiles of the target organs during systemic $C$. albicans infection were measured by cytometric bead array and ELISA analysis. The results demonstrated that interleukin-6 (IL-6) and IL-2 were statistically significant $(\mathrm{P}<0.05)$ in the spleen at 24 and $72 \mathrm{~h}$ post-infection, whereas in the kidney, IL-6 and tumor necrosis factor- $\alpha(\mathrm{TNF}-\alpha)$ were statistically significant $(\mathrm{P}<0.05)$ at 24 and $72 \mathrm{~h}$ post-infection and CXCL-1 and transforming growth factor- $\beta$ (TGF- $\beta)$ were statistically significant $(\mathrm{P}<0.05)$ at $72 \mathrm{~h}$ post-infection. In the brain, IL-6 and TNF- $\alpha$ were statistically significant $(\mathrm{P}<0.05)$ at 24 and $72 \mathrm{~h}$ post-infection, whereas TGF- $\beta$ was statistically significant $(\mathrm{P}<0.05)$ at $72 \mathrm{~h}$ post-infection. These findings demonstrate that host immune responses were varied among target organs during systemic C. albicans infection. This could be important for designing targeted immunotherapy against this pathogen through immunomodulatory approaches in future exploratory research.
\end{abstract}

Correspondence to: Mr. Voon Kin Chin or Dr Pei Pei Chong, Department of Biomedical Science, Faculty of Medicine and Health Sciences, University Putra Malaysia, Serdang, Selangor 43400, Malaysia

E-mail: cvk717@gmail.com

E-mail: cpp@upm.edu.my

Key words: Candida albicans, cytometric bead array analysis, ELISA, cytokines

\section{Introduction}

Cytokines are a group of low molecular weight proteins that act as a mediator between cells. They are produced by leukocytes and a variety of non-immune cells in the body in response to stimuli. Cytokines are messengers of the immune system and play critical roles in regulating the immune response, hematopoietic development and cell-to-cell communication, as well as host responses to infectious agents and inflammatory stimuli $(1,2)$.

Cytokines are not typically stored as preformed proteins. They are only produced when required in immune responses. Therefore, under normal circumstances, cytokines are not detectable or are present at low levels in body fluids or tissues. Their syntheses are initiated by gene transcription and their mRNAs are short lived. Fundamentally, their presence at elevated levels of expression or dysregulated production may associate with inflammation or disease pathogenesis (3).

Candida bloodstream infections remain the most frequent life-threatening fungal disease with Candida albicans (C. albicans) accounting for 70-80\% of the Candida isolates recovered from infected patients. Previously, life-threatening Candida infection continues to increase due to the existence of drug resistance in Candida, inefficacy of the available antifungal drugs, diagnostic procedures and a steady increase of immunocompromised patients (4).

Systemic infection with $C$. albicans often results in high mortality and morbidity rate in immunocompromised patients. During systemic candidiasis, C. albicans is carried by the bloodstream to almost all the organs of the body, with the immune responses occurring in the kidney influencing the C. albicans infection outcome (5-7). The brain is the second most affected organ. However, its immunopathogenesis remains incomplete and requires further study. The spleen is a primary lymphoid organ that may possess specific protective mechanisms to confer it from infection by $C$. albicans. In addition, previous studies have mostly centred on investigating the responses on kidney or a single host cell type, such as monocytes and neutrophiles, which do not reflect the real 
situation occurring in the affected organs undergoing C.albicans infection (8-10). Therefore, the aim of the present study was to assess the local cytokine production in various organs (spleen, kidney and brain) during systemic C. albicans infection, as local cytokine levels and activity are of considerably greater value for monitoring the pathological events in a target tissue, rather than systemic cytokine levels (11).

\section{Materials and methods}

Ethics statement. All the animal experiments were performed according to the guidelines in the Guide for the Care and Use of Laboratory Animals of the University Putra Malaysia Health Campus Animal Ethics Committee (Serdang, Selangor, Malaysia) and approved by the AnimalCare and UseCommittee, Faculty of Medicine and Health Sciences, University Putra Malaysia (UPM/FPSK/PADS/BR/UUH/00486).

Generation of acute systemic candidiasis in BALB/c mice. Six-week-old female BALB/c mice (weighing 20-25 g) were used for all the animal experiments. The animals were randomized and were provided food and water ad libitum. C. albicans cell inocula were prepared from a 24-h culture Sabouraud Dextrose Broth (SDB at $37^{\circ} \mathrm{C}$ ), which had been washed twice and re-suspended in phosphate-buffered saline (PBS) at the required density by using an Improved Neubauer haematocytometer (Camlab, Ltd., Cambridge, UK). Female $\mathrm{BALB} / \mathrm{c}$ mice were challenged intravenously through tail-vein injection of a $200-\mu 1$ inoculum of $C$. albicans $\left(5 \times 10^{5}\right.$ organisms/mouse), via a 27-gauge syringe. In the control group, $200 \mu 1$ PBS was used instead of the yeast suspension. For flow cytometry and ELISA analysis, a total of 18 mice were used and they were randomly assigned to 3 groups of 6 mice each, which were the uninfected, infected with $C$. albicans at $24 \mathrm{~h}$ post-infection and infected with $C$. albicans at $72 \mathrm{~h}$ post-infection groups.

Preparation of tissue homogenate supernatants. The female BALB/c mice were sacrificed at 24 and $72 \mathrm{~h}$ post-infection. Kidneys, spleen and brain organs were harvested and weighed. The organs were transferred to a microcentrifuge tube containing $0.8 \mathrm{ml}$ PBS and protease inhibitor (cOmplete ULTRA Tablets, Mini, EDTA-free; Roche Diagnostics, Mannheim, Germany). The mixtures were homogenized by a handheld homogenizer (Wiggen Hauser, Berlin, Germany) and the supernatants were collected from the mixture following spinning down and stored at $-80^{\circ} \mathrm{C}$ prior to analysis.

Flow cytometry analysis. Analysis of cytokines from the homogenates were conducted by the Mouse Th1/Th2/Th17 Cytometric Bead Array kit (CBA; BD Biosciences, Franklin Lakes, NJ, USA) as per the manufacturer's instructions and was analyzed on BD FACSCanto II flow cytometer (BD Biosciences). Standard curves were determined for each cytokine for a range of $20-5,000 \mathrm{pg} / \mathrm{ml}$. The following cytokines were measured: Interleukin-6 (IL-6), IL-10, interferon- $\gamma$ (IFN- $\gamma$ ), tumor necrosis factor- $\alpha$ (TNF- $\alpha)$, IL-2, IL-4 and IL-17a. The results for the standard curves of each cytokines and samples were generated using FCAP Array software v3.0 (BD Biosciences).
ELISA analysis. The protein expression level of keratinocyte-derived chemokine (CXCL-1/KC; NovaTeinBio, Boston, MA, USA) and transforming growth factor- $\beta$ (TGF- $\beta$; NovaTeinBio) in the brain, kidneys and spleen at $72 \mathrm{~h}$ post-infection were assayed by ELISA according to the manufacturer's instructions. Standard curves were determined for TGF- $\beta$ for a range of $2.5-80 \mathrm{ng} / \mathrm{ml}$ and CXCL-1/KC for a range of $15.6-1,000 \mathrm{pg} / \mathrm{ml}$. The optical densities were measured at $450 \mathrm{~nm}$ using a SpectraMax 190 spectrophotometer (Beckman Coulter, Brea, CA, USA). The ELISAs were performed in duplicates. The detection limit for this assay was $2.5 \mathrm{ng} / \mathrm{ml}$ for TGF- $\beta$ and $15.6 \mathrm{pg} / \mathrm{ml}$ for CXCL-1/KC. The concentrations of the samples were calculated by fitting the optical density values of each sample into the equation generated from the standard curve graph.

Statistical analysis. The protein concentration of each cytokine measured: IL-6, IL-10, IFN- $\gamma$, TNF- $\alpha$, IL-2, IL-4, IL-17a, CXCL-1/KC and TGF- $\beta$; at 24 and $72 \mathrm{~h}$ post-infection was compared to the uninfected group and was analyzed by Mann-Whitney $\mathrm{U}$ test. $\mathrm{P}<0.05$ was considered to indicate a statistically significant difference.

\section{Results}

Evaluation of cytokine production in kidney, spleen and brain homogenates. Analysis of the cytokines in the tissue homogenates of 6 mice from infected mice with $C$. albicans and control mice was carried out by the CBA technique, which allowed the simultaneous measurement of several cytokines in a small volume of samples. Seven cytokines (IL-2, IL-4, IL-6, IL-10, TNF- $\alpha$, IFN- $\gamma$ and IL-17a) were measured and the results are shown in Tables I-III. In the kidney, IL-6 and TNF- $\alpha$ concentrations were statistically increased $(\mathrm{P}<0.05)$ at $24 \mathrm{~h}$ post-infection, whereas IL-10, IL-17a and IL-2 concentrations were increased and IFN- $\gamma$ and IL- 4 concentrations were reduced, however, not to a significant extent at $24 \mathrm{~h}$ post-infection. IL-6 and TNF- $\alpha$ concentrations were statistically increased $(\mathrm{P}<0.05)$ at $72 \mathrm{~h}$ post-infection, whereas IFN- $\gamma$ and IL-17a concentrations were increased and IL-10, IL-2 and IL-4 concentrations were reduced but not to a significant extent at $72 \mathrm{~h}$ post-infection.

In the brain, IL- 6 and TNF- $\alpha$ concentrations were statistically increased $(\mathrm{P}<0.05)$ at $24 \mathrm{~h}$ post-infection, whereas IFN- $\gamma$, IL-2 and IL-4 concentrations were increased but not to a significant extent at $24 \mathrm{~h}$ post-infection. IL-17a was not detected in the brain at $24 \mathrm{~h}$ post-infection. IL- 6 and TNF- $\alpha$ concentrations were statistically increased $(\mathrm{P}<0.05)$ at $72 \mathrm{~h}$ post-infection, whereas IL-10, IL-2 and IL-17a concentrations were reduced but not to a significant extent at $72 \mathrm{~h}$ post-infection. IFN- $\gamma$ and IL- 4 were not detected in the brain at $72 \mathrm{~h}$ post-infection.

In the spleen, IL-6 and IL-2 concentrations were statistically increased $(\mathrm{P}<0.05)$ at $24 \mathrm{~h}$ post-infection, whereas IL-4, TNF- $\alpha$, IL-10 and IL-17a concentrations were increased and the IFN- $\gamma$ concentration was reduced but not to a significant extent at $24 \mathrm{~h}$ post-infection. IL-6 and IL-2 concentrations were statistically increased $(\mathrm{P}<0.05)$ at $72 \mathrm{~h}$ post-infection, whereas IL-2 and IL-17a concentrations were increased and IL-10 and TNF- $\alpha$ concentrations were reduced but not to a 
Table I. Cytokine concentrations in the kidney of uninfected mice and mice infected systemically with C.albicans at 24 and $72 \mathrm{~h}$ post-infection by cytometric bead array analysis.

\begin{tabular}{|c|c|c|c|c|c|}
\hline \multirow[b]{2}{*}{ Kidney } & \multirow{2}{*}{$\begin{array}{l}\text { Uninfected group, } \\
\text { mean } \pm \text { SEM }\end{array}$} & \multicolumn{2}{|c|}{24 h post-infection with $C$. albicans } & \multicolumn{2}{|c|}{72 h post-infection with $C$. albicans } \\
\hline & & Mean \pm SEM & P-value & Mean \pm SEM & P-value \\
\hline IFN- $\gamma$ & $9.06 \pm 2.35$ & $3.18 \pm 2.13$ & 0.115 & $11.90 \pm 2.04$ & 0.334 \\
\hline IL-10 & $137.90 \pm 34.85$ & $144.50 \pm 37.73$ & 1.000 & $95.16 \pm 33.63$ & 0.470 \\
\hline IL-17a & $14.13 \pm 1.61$ & $18.17 \pm 5.47$ & 0.590 & $16.59 \pm 2.31$ & 0.520 \\
\hline IL-2 & $21.40 \pm 1.82$ & $21.58 \pm 5.10$ & 0.570 & $20.62 \pm 4.70$ & 0.630 \\
\hline IL-4 & $15.47 \pm 5.34$ & $6.98 \pm 3.60$ & 0.140 & $12.69 \pm 5.86$ & 0.280 \\
\hline IL-6 & $19.46 \pm 4.67$ & $265.90 \pm 44.28$ & 0.002 & $6513.00 \pm 3356.00$ & 0.002 \\
\hline TNF- $\alpha$ & $34.26 \pm 10.00$ & $549.90 \pm 53.39$ & 0.002 & $3551.00 \pm 1418.00$ & 0.002 \\
\hline
\end{tabular}

Results are expressed as mean \pm standard error of the mean (SEM) and the concentration of each cytokine measured is expressed in pg/g of kidney tissues. $\mathrm{P}<0.05$ was considered to indicate a statistically significant difference. C. albicans, Candida albicans; IFN- $\gamma$, interferon- $\gamma ; \mathrm{IL}$, interleukin; TNF- $\alpha$, tumor necrosis factor- $\alpha$.

Table II. Cytokine concentrations in the spleen of uninfected mice and mice infected systemically with C.albicans at 24 and $72 \mathrm{~h}$ post-infection by cytometric bead array analysis.

\begin{tabular}{|c|c|c|c|c|c|}
\hline \multirow[b]{2}{*}{ Spleen } & \multirow{2}{*}{$\begin{array}{l}\text { Uninfected group, } \\
\text { mean } \pm \text { SEM }\end{array}$} & \multicolumn{2}{|c|}{24 h post-infection with $C$. albicans } & \multicolumn{2}{|c|}{$72 \mathrm{~h}$ post-infection with $C$. albicans } \\
\hline & & Mean \pm SEM & P-value & Mean \pm SEM & P-value \\
\hline IFN- $\gamma$ & $3.80 \pm 1.29$ & $3.33 \pm 1.68$ & 0.796 & $0.00 \pm 0.00$ & 0.334 \\
\hline IL-10 & $21.03 \pm 13.35$ & $33.26 \pm 15.7$ & 0.591 & $18.46 \pm 12.31$ & 0.924 \\
\hline IL-17a & $3.45 \pm 2.70$ & $7.51 \pm 0.46$ & 0.060 & $7.13 \pm 1.64$ & 0.158 \\
\hline IL-2 & $5.98 \pm 2.86$ & $18.07 \pm 2.19$ & 0.030 & $17.15 \pm 2.54$ & 0.028 \\
\hline IL-4 & $1.43 \pm 1.43$ & $5.81 \pm 3.74$ & 0.462 & $0.61 \pm 0.61$ & 1.000 \\
\hline IL-6 & $1.83 \pm 1.83$ & $16.11 \pm 1.08$ & 0.004 & $102.70 \pm 38.27$ & 0.004 \\
\hline TNF- $\alpha$ & $65.29 \pm 15.95$ & $70.54 \pm 5.83$ & 1.000 & $30.73 \pm 9.46$ & 0.180 \\
\hline
\end{tabular}

Results are expressed in mean \pm standard error of the mean (SEM) and the concentration of each cytokine measured is expressed in pg/g of spleen tissues. $\mathrm{P}<0.05$ was considered to indicate a statistically significant difference. C. albicans, Candida albicans; IFN- $\gamma$, interferon- $\gamma ;$ IL, interleukin; TNF- $\alpha$, tumor necrosis factor- $\alpha$.

Table III. Cytokine concentrations in the brain of uninfected mice and mice infected systemically with C.albicans at 24 and $72 \mathrm{~h}$ post-infection by cytometric bead array analysis.

\begin{tabular}{|c|c|c|c|c|c|}
\hline \multirow[b]{2}{*}{ Brain } & \multirow{2}{*}{$\begin{array}{l}\text { Uninfected group } \\
\text { mean } \pm \text { SEM }\end{array}$} & \multicolumn{2}{|c|}{24 h post-infection with $C$. albicans } & \multicolumn{2}{|c|}{72 h post-infection with $C$. albicans } \\
\hline & & Mean \pm SEM & P-value & Mean \pm SEM & P-value \\
\hline IFN- $\gamma$ & $1.33 \pm 1.33$ & $5.68 \pm 2.64$ & 0.182 & $1.33 \pm 1.33$ & 0.902 \\
\hline IL-10 & $80.87 \pm 45.72$ & $162.20 \pm 2.48$ & 0.170 & $25.66 \pm 16.26$ & 0.532 \\
\hline IL-17a & $7.39 \pm 2.47$ & $1.54 \pm 1.54$ & 0.090 & $2.54 \pm 1.61$ & 0.104 \\
\hline IL-2 & $17.28 \pm 3.23$ & $20.28 \pm 4.78$ & 0.057 & $7.98 \pm 3.60$ & 0.060 \\
\hline IL-4 & $0.00 \pm 0.00$ & $9.25 \pm 4.41$ & 0.462 & $0.00 \pm 0.00$ & 1.000 \\
\hline IL-6 & $2.72 \pm 2.72$ & $88.53 \pm 8.32$ & 0.004 & $747.90 \pm 500.40$ & 0.004 \\
\hline TNF- $\alpha$ & $3.61 \pm 3.61$ & $69.53 \pm 13.40$ & 0.006 & $1570 \pm 584.50$ & 0.004 \\
\hline
\end{tabular}

Results are expressed as mean \pm standard error of the mean (SEM) and the concentration of each cytokine measured is expressed in pg/g of brain tissues. $\mathrm{P}<0.05$ was considered to indicate a statistically significant difference. C. albicans, Candida albicans; IFN- $\gamma$, interferon- $\gamma ;$ IL, interleukin; TNF- $\alpha$, tumor necrosis factor- $\alpha$. 
Table IV. Cytokine concentrations in the kidney, spleen and brain homogenates of uninfected mice and mice infected systemically with $C$. albicans at $72 \mathrm{~h}$ post-infection by ELISA analysis.

\begin{tabular}{lccc}
\hline & & & \\
Uninfected, & h post-infection & \\
Organ & mean \pm SEM & albicans, & \\
mean \pm SEM & P-value \\
\hline Kidney & & & \\
TGF- $\beta$ & $201.40 \pm 6.75$ & $285.20 \pm 7.52$ & 0.002 \\
CXCL-1 & $1056.00 \pm 160.00$ & $1942.00 \pm 206.90$ & 0.015 \\
Spleen & & & \\
TGF- $\beta$ & $155.00 \pm 9.74$ & $175.50 \pm 10.97$ & 0.240 \\
CXCL-1 & $380.80 \pm 21.61$ & $368.10 \pm 21.31$ & 0.818 \\
Brain & & & \\
TGF- $\beta$ & $217.50 \pm 6.97$ & $271.70 \pm 2.58$ & 0.002 \\
CXCL-1 & $484.10 \pm 26.71$ & $462.70 \pm 44.07$ & 0.699 \\
\hline
\end{tabular}

Results are expressed as mean \pm standard error of the mean (SEM). Cytokine concentration of TGF- $\beta$ (ng/g) and CXCL- 1 (pg/g) in kidney, spleen and brain tissues. $\mathrm{P}<0.05$ was considered to indicate a statistically significant difference. $C$. albicans, Candida albicans; TGF- $\beta$, transforming growth factor- $\beta$.

significant extent at $72 \mathrm{~h}$ post-infection. IFN- $\gamma$ and IL-4 were not detected in the spleen at $72 \mathrm{~h}$ post-infection.

The concentrations of TGF- $\beta$ and CXCL- $1 / \mathrm{KC}$ were also measured in the kidney, spleen and brain homogenates at $72 \mathrm{~h}$ post-infection and the result is shown in Table IV. In the kidney, TGF- $\beta$ and CXCL-1/KC concentrations were statistically increased $(\mathrm{P}<0.05)$ at $72 \mathrm{~h}$ post-infection, whereas in the brain, the TGF- $\beta$ concentration was statistically increased $(\mathrm{P}<0.05)$ and $\mathrm{CXCL}-1 / \mathrm{KC}$ was increased but not to a significant extent at $72 \mathrm{~h}$ post-infection. By contrast, in the spleen the TGF- $\beta$ concentration was increased, whereas the CXCL-1/KC concentration was reduced but not to a significant extent at $72 \mathrm{~h}$ post-infection.

\section{Discussion}

Since cytokine polarization is involved in a number of pathological conditions, profiling of several cytokines may be more beneficial than measuring one cytokine in a single sample. Flow cytometric multiplexed bead assays offer numerous advantages in measuring soluble cytokines. Multiple analytes can be measured simultaneously in a single sample volume and standard curves can be generated for all the analytes from one standard mixture. This in turn reduces analytical time, effort and cost when compared to the existing methods. The use of fluorescence-based measurement may provide more sensitivity and a broader range for cytokine quantification compared to colorimetric measurements $(12,13)$.

The present study utilized a clinical isolate of $C$. albicans isolated from the vaginal site of a healthy individual in Malaysia to investigate how $C$. albicans, as a commensal, can cause life threatening systemic infection in a debilitated host in the intravenous challenge murine model. In addition, usage of clinical $C$. albicans from a particular region could be of importance and useful for investigation the causal association between the severity of candidiasis in that particular region and the yeast strain used, and therefore, can be documented for evaluation in later stage.

In our previous study, higher yeast loads were found in the kidney, brain and spleen during $72 \mathrm{~h}$ of post-infection (unpublished data). Additionally, the gene array results demonstrated that there was continued expression of local host immune genes in the kidney and brain over $72 \mathrm{~h}$ post-infection, while the spleen showed minimal expression with the majority of the local host immune genes suppressed over $72 \mathrm{~h}$ post-infection (unpublished data). Therefore, the present study aimed to profile the protein expression of local cytokines in the kidney, brain and spleen to gain more insight on how these cytokines interact during systemic $C$. albicans infection.

In the study, the kinetics of the cytokines produced over 72 h post-infection with $C$. albicans were demonstrated and the findings indicated that production of cytokines may be dependent on tissue type. Different organs showed different cytokine phenotypes during systemic $C$. albicans infection through CBA and ELISA analysis. At $24 \mathrm{~h}$ post-infection, the innate response that mainly involves IL-6 and TNF- $\alpha$ was observed in the kidney and brain, whereas the innate/Th responses, which mainly involve IL-6 and IL-2, were observed in the spleen. At $72 \mathrm{~h}$ post-infection, the innate/suppressive responses, which mainly involve IL- 6 , TNF- $\alpha$, TGF- $\beta$ and CXCL-1, were observed in the kidney. The innate/Th responses, which mainly involve IL-6 and IL-2, were observed in the spleen and the innate/suppressive responses, which mainly involve IL-6, TNF- $\alpha$ and TGF- $\beta$, were observed in brain.

Previous studies have demonstrated that resistance to systemic candidiasis is associated with development of the Th1 response, which is on the basis on IFN- $\gamma$ secretion (14), whereas a fatal outcome is linked to the development of Th2 response, which is on the basis of IL-4, IL-5 and IL-13 secretion and IL-10 responses in kidney $(5,6)$. However, in the present study, there were no significant changes of IFN- $\gamma$, IL-4 and IL-10 concentrations in the kidney, spleen and brain homogenates infected with C. albicans, which could be due to differences in yeast strain used and kinetics of study. Furthermore, the lack of production of the Th1 response in the brain and kidney in the study was supported by the lack of IL-2 cytokine, a T-cell derived cytokine.

Previously, the Th17 cell, which is on the basis of the IL-17 cytokine family, has importance in inflammatory and autoimmune diseases $(15,16)$. A previous study has shown the involvement of the Th17 response in systemic candidiasis and the Th17 response has a clear protective effect in disseminated murine $C$. albicans infection, where IL-17a receptor-knockout mice resulted in reduced survival and increased fungal burden in the kidneys (17). In the present study, the IL-17a concentrations in the kidney and spleen were higher than the uninfected group, but IL-17a was not detected in the brain homogenate. This indicated that IL-17a may be involved in regulating the systemic $C$. albicans in the kidney and spleen, but not in the brain. This in turn suggested that IL-17a production may be tissue dependant during systemic candidiasis.

The presence of TGF- $\beta$ in the kidney and brain, but not in the spleen, at $72 \mathrm{~h}$ post-infection indicated that this cytokine was involved in promoting disease progression in these organs 
during systemic $C$. albicans infection. TGF- $\beta$ is an inhibitory cytokine $(18,19)$ that suppresses phagocyte function in mice infected with $C$. albicans. TGF- $\beta$ downregulates activated monocytes and macrophages, suppressing IFN- $\gamma$-induced production of nitric oxide (20), which would favour the dissemination and progression of $C$. albicans infection (21). This demonstrated that the local production of active TGF- $\beta$ by hepatocytes and by infected mononuclear cells is a component of the response to $C$. albicans infection that most possibly contributes to disease progression in the immunocompromised host. By contrast, active TGF- $\beta$ suppresses microbicidal activity against Leishmania or Trypanosoma cruzi infection and enhances the proliferation of the pathogen (22-24).

The presence of IL-2 in the spleen may protect it from infection by $C$. albicans. IL-2 is a proinflammatory cytokine produced by activated $\mathrm{T}$ cells and it is necessary for the generation of an optimal cell-mediated immune response. A previous study showed that IL-2-activated lymphocytes can limit the growth of $C$. albicans hyphae (25). In another study, IL-2 was demonstrated to activate human lymphocytes to attack and inhibit the growth of Cryptococcus neoformans. Furthermore, IL-2-activated T cells can attack and mediate anti-cryptococcal activity without MHC restriction (26).

CXCL-1 is involved in the mobilization of leukocyte infiltrates, particularly neutrophil, towards the site of infection and is transcriptionally regulated by signalling through toll-like receptor (TLR) 2, 3 and 4 (27). In the present study, CXCL-1 was statistically increased in the kidney only at $72 \mathrm{~h}$ post-infection. In addition (7), $\mathrm{KC}$ production is considered to be a critical early event that mobilizes the host infiltrates that appear in the kidney. Therefore, in agreement with previous studies, we believe that CXCL-1 is an important mediator in regulating the systemic $C$. albicans infection locally, particularly in the kidney.

Although inflammatory responses are a dominant feature of the early response to $C$. albicans, these are under precise control in order to avoid excessive tissue damage. The result of the present study demonstrated that TNF- $\alpha$ concentration was extremely high in the brain and kidney. TNF- $\alpha$ enhances the anti-candidal function of macrophages and neutrophils to kill $C$. albicans directly $(28,29)$ and is a key factor in the reduction of the pathogenic burden of $C$. albicans in normal and immunosuppressed animals. The low levels of TNF- $\alpha$ play a protective role by inducing macrophages to produce microbicidal reactive intermediates and natural killer cells to produce IFN- $\gamma$ (30). However, high levels of TNF- $\alpha$ may increase the sensitivity of infection in the brain. Susceptibility to cerebral malaria in mice has been associated with the Th1 or high TNF- $\alpha$ responses $(31,32)$. Furthermore, the TNF- $\alpha$ level increased in the serum of children with uncomplicated Plasmodium falciparum malaria, but markedly increased in children with a fatal outcome from cerebral malaria, leading to speculation that excessive levels of TNF- $\alpha$ production may predispose cerebral malaria and a fatal outcome (33).

A high level of TNF- $\alpha$ may lead to amplification of immune responses to infection in the kidney, as TNF- $\alpha$ has been shown to induce renal expression of TLR2 and 4 (34), and continual renal expression of TLR 2 and 4 lead to continued production of proinflammatory and immunosuppressive mediators in response to fungal cells. A high level of TNF- $\alpha$ is associated with organ failure and septic shock, which can lead to host deterioration and eventually death in the mouse model of systemic $C$. albicans infection $(6,35)$.

By contrast, the present study found that IL- 6 concentration was high in kidneys infected with $C$. albicans as compared to uninfected control. A previous study has shown that IL-6 expression is associated with the recruitment of neutrophils to the site of infection in mice. IL-6 deficiency, resulting from gene targeting, increases mouse susceptibility to $C$. albicans infection and prevents development of protective Thl-mediated immunity (36). Van Snick 1990 demonstrated that limited IL-6 production was found in conventional mice with healing infection of the yeast and indicates that IL- 6 production must be strictly regulated to avoid immunopathological consequences from its deregulation (37). In the neonatal mouse model infected with enterovirus 71, sustained high levels of IL-6 production lead to severe tissue damage and eventually death of the animals (38). In another study by Tanaka et al (39), overexpression of IL-6 promotes myocardial injury by interrupting the cytokine network and viral clearance and indicated that IL-6 is one factor that accelerates tissue damage in the viral myocarditis. Therefore, a high IL- 6 concentration found in the kidneys of mice infected with $C$. albicans may contribute to disease progression and damage to the tissues.

There are certain limitations in the present study, such as use of a single strain of $C$. albicans isolated from an immunocompetent patient, the protein profiling was limited to a targeted group of cytokines and the kinetics of the study was too short. In future studies, other inflammatory and non-inflammatory biomarkers that were not included in the present analysis should be investigated, and the subset(s) of cells that are responsible for the expression of immune response towards systemic C. albicans infection should be identified.

In conclusion, the early host defense against systemic C. albicans lies in the innate ability to control the yeast growth in respective target organs that allow subsequent development of adaptive immunity. The differential profiles of the cytokines that appear in the kidney, spleen and brain indicated that the demonstrated local host immune responses were varied among the organs during systemic $C$. albicans infection and this could have crucial implications for future work of targeted therapies using immunomodulatory approaches.

\section{Acknowledgements}

The authors are grateful to University Putra Malaysia for the financial support through RUGS grant (project nos. 04-01-12-1607RU and 04-02-12-1761RU).

\section{References}

1. Dinarello CA: Proinflammatory cytokines. Chest 118: 503-508, 2000.

2. Meager T (ed): The Molecular Biology of Cytokines. John Wiley \& Sons, Chichester, UK, pp1-21, 1998.

3. Abbas AK, Lichtman AH and Pober JS (eds): Cellular and Molecular Immunology. 3rd edition. W.B. Saunders, Philadelphia, PA, pp15-37, 1997.

4. Fernández-Arenas E, Cabezón V, Bermejo C, Arroyo J, Nombela C, Diez-Orejas R and Gil C: Integrated proteomics and genomics strategies bring new insight into Candida albicans response upon macrophage interaction. Mol Cell Proteomics 6: 460-478, 2007. 
5. Brieland J,Essig D, Jackson C, Frank D, Loebenberg D, Menzel F, Arnold B, DiDomenico B and Hare R: Comparison of pathogenesis and host immune responses to Candida glabrata and Candida albicans in systemically infected immunocompetent mice. Infect Immun 69: 5046-5055, 2001.

6. Spellberg B, Ibrahim AS, Edwards JE Jr and Filler SG: Mice with disseminated candidiasis die of progressive sepsis. J Infect Dis 192: 336-343, 2005.

7. MacCallum DM, Castillo L, Brown AJ, Gow NA and Odds FC: Early-expressed chemokines predict kidney immunopathology in experimental disseminated Candida albicans infections. PLoS One 4: e6420, 2009.

8. Fradin C, De Groot P, MacCallum D, Schaller M, Klis F, Odds FC and Hube B: Granulocytes govern the transcriptional response, morphology and proliferation of Candida albicans in human blood. Mol Microbiol 56: 397-415, 2005

9. MacCallum DM: Massive induction of innate immune response to Candida albicans in the kidney in a murine intravenous challenge model. FEMS Yeast Res 9: 1111-1122, 2009.

10. Kim HS, Choi EH, Khan J, Roilides E, Francesconi A, Kasai M, Sein T, Schaufele RL, Sakurai K, Son CG, Greer BT, Chanock S, Lyman CA and Walsh TJ: Expression of genes encoding innate host defense molecules in normal human monocytes in response to Candida albicans. Infect Immun 73: 3714-3724, 2005.

11. Mathey E, Pollard J and Armati P: In situ hybridization for cytokines in human tissue biopsies. Methods Mol Biol 249: 41-46, 2003.

12. Edwards BS, Oprea T, Prossnitz ER and Sklar LA: Flow cytometry for high-throughput, high-content screening. Curr Opin Chem Biol 8: 392-398, 2004.

13. Diaz MR, Boekhout T, Theelen B, Bovers M, Cabañes FJ and Fell JW: Microcoding and flow cytometry as a high-throughput fungal identification system for Malassezia species. J Med Microbiol 55: 1197-1209, 2006.

14. Ashman RB and Papadimitriou JM: Production and function of cytokines in natural and acquired immunity to Candida albicans infection. Microbiol Rev 59: 646-672, 1995.

15. Moseley TA, Haudenschild DR, Rose L and Reddi AH: Interleukin-17 family and IL-17 receptors. Cytokine Growth Factor Rev 14: 155-174, 2003

16. Kolls JK and Lindén A: Interleukin-17 family members and inflammation. Immunity 21: 467-476, 2004.

17. Huang W, Na L, Fidel PL and Schwarzenberger P: Requirement of interleukin-17A for systemic anti-Candida albicans host defense in mice. J Infect Dis 190: 624-631, 2004.

18. Blobe GC, Schiemann WP and Lodish HF: Role of transforming growth factor beta in human disease. N Engl J Med 342: 1350-1358, 2000.

19. Letterio JJ and Roberts AB: Regulation of immune responses by TGF-beta. Annu Rev Immunol 16: 137-161, 1998.

20. Vodovotz Y, Bogdan C, Paik J, Xie QW and Nathan C: Mechanisms of suppression of macrophage nitric oxide release by transforming growth factor beta. J Exp Med 178: 605-613, 1993.

21. Letterio JJ, Lehrnbecher T, Pollack G, Walsh TJ and Chanock SJ: Invasive candidiasis stimulates hepatocyte and monocyte production of active transforming growth factor beta. Infect Immun 69: 5115-5120, 2001.

22. Silva JS, Twardzik DR and Reed SG: Regulation of Trypanosoma cruzi infections in vitro and in vivo by transforming growth factor beta (TGF-beta). J Exp Med 174: 539-545, 1991.

23. Barral A, Barral-Netto M, Yong EC, Brownell CE, Twardzik DR and Reed SG: Transforming growth factor beta as a virulence mechanism for Leishmania braziliensis. Proc Natl Acad Sci USA 90: 3442-3446, 1993.

24. Gorelik L and Flavell RA: Abrogation of TGFbeta signaling in $\mathrm{T}$ cells leads to spontaneous $\mathrm{T}$ cell differentiation and autoimmune disease. Immunity 12: 171-181, 2000.
25. Beno DW and Mathews HL: Growth inhibition of Candida albicans by interleukin-2-activated splenocytes. Infect Immun 60: 853-863, 1992.

26. Levitz SM and Dupont MP: Phenotypic and functuional characterization of human lymphocytes activated by interleukin-2 to directly inhibit growth of Crytococcus neoformans in vitro. J Clin Invest 91: 1490-1498, 1993.

27. De Filippo K, Henderson RB, Laschinger M and Hogg N: Neutrophil chemokines KC and macrophage-inflammatory protein-2 are newly synthesized by tissue macrophages using distinct TLR signaling pathways. J Immunol 180: 4308-4315, 2008.

28. Djeu JY, Blanchard DK, Halkis D and Friedman H: Growth inhibition of Candida albicans by human polymorphonuclear neutrophils: activation by interferon-gamma and tumor necrosis factor. J Immunol 137: 2980-2984, 1986.

29. Ferrante A: Tumor necrosis factor alpha potentiates neutrophil antimicrobial activity: increased fungicidal activity against Torulopsis glabrata and Candida albicans and associated increases in oxygen radical production and lysosomal enzyme release. Infect Immun 57: 2115-2122, 1989.

30. Marshall JD, Heeke DS, Abbate C, Yee P and Van Nest G: Induction of interferon-gamma from natural killer cells by immunostimulatory $\mathrm{CpG}$ DNA is mediated through plasmacytoid-dendritic-cell-produced interferon-alpha and tumour necrosis factor-alpha. Immunology 117: 38-46, 2006.

31. Grau GE, Heremans H, Piguet PF, Pointaire P, Lambert PH, Billiau A and Vassalli P: Monoclonal antibody against interferon gamma can prevent experimental cerebral malaria and its associated overproduction of tumor necrosis factor. Proc Natl Acad Sci USA 86: 5572-5574, 1989.

32. de Kossodo S and Grau GE: Profiles of cytokine production in relation with susceptibility to cerebral malaria. J Immunol 151: 4811-4820, 1993.

33. Kwiatkowski D, Hill AV, Sambou I, Twumasi P, Castracane J, Manogue KR, Cerami A, Brewster DR and Greenwood BM: TNF concentration in fatal cerebral, non-fatal cerebral, and uncomplicated Plasmodium falciparum malaria. Lancet 336: 1201-1204, 1990

34. Wolfs TG, Buurman WA, van Schadewijk A, de Vries B, Daemen MA, Hiemstra PS and van't Veer C: In vivo expression of Toll-like receptor 2 and 4 by renal epithelial cells: IFN-gamma and TNF-alpha mediated up-regulation during inflammation. J Immunol 168: 1286-1293, 2002.

35. Mencacci A, Cenci E, Del Sero G, Fe d'Ostiani C, Mosci P, Montagnoli C, Bacci A, Bistoni F, Quesniaux VF, Ryffel B and Romani L: Defective co-stimulation and impaired Th1 development in tumor necrosis factor/lymphotoxin-alpha double-deficient mice infected with Candida albicans. Int Immunol 10: 37-48, 1998.

36. Romani L, Mencacci A, Cenci E, Spaccapelo R, Toniatti C, Puccetti P, Bistoni F and Poli V: Impaired neutrophil response and CD4+ T helper cell 1 development in interleukin 6-deficient mice infected with Candida albicans. J Exp Med 183: 1345-1355, 1996.

37. Van Snick J: Interleukin-6: an overview. Annu Rev Immunol 8: 253-278, 1990

38. Khong WX, Foo DG, Trasti SL, Tan EL and Alonso S: Sustained high levels of interleukin- 6 contribute to the pathogenesis of enterovirus 71 in a neonate mouse model. J Virol 85: 3067-3076, 2001.

39. Tanaka T, Kanda T, McManus BM, Kanai H, Akiyama H, Sekiguchi K, Yokoyama T and Kurabayashi M: Overexpression of interleukin- 6 aggravates viral myocarditis: impaired increase in tumor necrosis factor-alpha. J Mol Cell Cardiol 33: 1627-1635, 2001. 